\title{
Optimal Matching for Prostate Brachytherapy Seed Localization with Dimension Reduction*
}

\author{
Junghoon Lee ${ }^{1}$, Christian Labat ${ }^{2}$, Ameet K. Jain ${ }^{3}$, Danny Y. Song ${ }^{4}$, \\ Everette C. Burdette $^{5}$, Gabor Fichtinger ${ }^{2,6}$, and Jerry L. Prince ${ }^{1}$ \\ 1 Department of Electrical and Computer Eng., Johns Hopkins University, USA \\ ${ }^{2}$ Department of Computer Science, Johns Hopkins University, USA \\ ${ }^{3}$ Philips Research North America, USA \\ 4 Department of Radiation Oncology, Johns Hopkins School of Medicine, USA \\ 5 Acoustic MedSystems, Inc., USA \\ 6 School of Computing, Queen's University, Canada
}

\begin{abstract}
In prostate brachytherapy, x-ray fluoroscopy has been used for intra-operative dosimetry to provide qualitative assessment of implant quality. More recent developments have made possible 3D localization of the implanted radioactive seeds. This is usually modeled as an assignment problem and solved by resolving the correspondence of seeds. It is, however, NP-hard, and the problem is even harder in practice due to the significant number of hidden seeds. In this paper, we propose an algorithm that can find an optimal solution from multiple projection images with hidden seeds. It solves an equivalent problem with reduced dimensional complexity, thus allowing us to find an optimal solution in polynomial time. Simulation results show the robustness of the algorithm. It was validated on 5 phantom and 18 patient datasets, successfully localizing the seeds with detection rate of $\geq 97.6 \%$ and reconstruction error of $\leq 1.2 \mathrm{~mm}$. This is considered to be clinically excellent performance.
\end{abstract}

\section{Introduction}

Low dose rate permanent brachytherapy is widely utilized for low risk prostate cancer, the success of which mainly depends on the ability to place an adequate number (50-120) of radioactive seeds to deliver a sufficient therapeutic dose distribution to the target gland while sparing adjacent organs from excessive radiation. During the procedure, the surgeon implants radioactive seeds based on a pre-operative implantation plan under transrectal ultrasound image guidance. However, it is not possible to accurately implant the seeds to the preplanned positions due to various reasons, e.g., patient motion, needle deflection within prostate, and edema. In order to improve outcomes and reduce complications, intra-operative localization of the seeds using x-ray fluoroscopy and intra-operative dosimetry modifications have been previously proposed [ $1 / 2] 3]$.

The seed localization problem from multiple fluoroscopy images are usually modeled as an assignment problem [3]. This approach resolves which segmented

\footnotetext{
^ This work has been supported by DoD PC050042 and NIH/NCI 2R44CA099374.
} 
seeds in each projection image correspond to the same physical seed. The 3D locations of the seeds are determined by computing so-called symbolic intersection of the lines connecting the segmented seeds to the x-ray source positions based on the revealed seed correspondence. However, this assignment problem is NP-hard [3. In addition, there exist a significant number of hidden seeds in every image, thus making explicit segmentation of seeds in every image hard. Such so-called hidden seeds are usually determined manually, and it is sometimes impossible to recover them when one seed completely hides another. Therefore, an algorithm that is computationally efficient and is able to solve the hidden seed problem is essential to a clinically feasible system.

There has been some research on solving the hidden seed problem. Su et al. 4] extended Fast-CARS [2], but the new algorithm was prone to reconstruct a greater number of seeds than were actually present, an effect called "false positive" seeds. Narayanan et al. [5] proposed a pseudo-seed-matching strategy coupled with an epipolar geometry-based reconstruction. This method requires at least one of the three images to be complete, however, or it may or may not reliably reconstruct the 3-D seed positions of the hidden seeds. Su et al. 6 proposed an adaptive grouping technique which divides the seed images into groups for efficient seed reconstruction and solving the hidden seed problem. Unfortunately, it may fail to detect overlapping seeds when the projection with the largest number of seed images among the divided groups is incomplete. Also, incorrect division of triplets may result in false positive seeds. Tomosynthesis 7 ] and Hough trajectory [8] methods have also been proposed, but they require unfeasibly large numbers of images in order to guarantee stable reconstruction.

We have previously proposed a dimension reduction approach for solving seed matching problem 9. However, it did not solve the hidden seed problem and was not computationally efficient. In this paper, we present an algorithm that is able to solve the hidden seed problem using dimensionality reduction to achieve efficient cost computation. We also propose a pruning algorithm that yields a dramatic reduction in computation time.

\section{Method}

\subsection{Extended Assignment Problem}

When at least three projection images are used and all the 2D seed locations are identified in every x-ray image, the correspondence problem can be formulated as a 3D assignment problem (3DAP) [3. In reality, however, there are a significant number of hidden seeds, resulting in a varying number of segmented seeds in each image. Here, we describe an extended assignment problem (EAP) that is able to reconstruct seed positions including hidden seeds.

In contrast to the 3DAP where exactly $N$ implanted seeds are identified in every image, we consider a different number $N_{i}$ of identified seeds in each image $i$ with $N_{i} \leq N$. For $I(\geq 3)$ x-ray images, the EAP is defined as:

$$
\min _{x_{i_{1} i_{2} i_{3} \ldots i_{I}}} \sum_{i_{1}=1}^{N_{1}} \sum_{i_{2}=1}^{N_{2}} \sum_{i_{3}=1}^{N_{3}} \cdots \sum_{i_{I}=1}^{N_{I}} c_{i_{1} i_{2} i_{3} \ldots i_{I}} x_{i_{1} i_{2} i_{3} \ldots i_{I}},
$$




$$
\text { s.t. }\left\{\begin{array}{l}
\sum_{i_{2}=1}^{N_{2}} \sum_{i_{3}=1}^{N_{3}} \cdots \sum_{i_{I}=1}^{N_{I}} x_{i_{1} i_{2} i_{3} \ldots i_{I}} \geq 1, \quad \forall i_{1} \\
\vdots \\
\sum_{i_{1}=1}^{N_{1}} \sum_{i_{2}=1}^{N_{2}} \cdots \sum_{i_{I-1}=1}^{N_{I-1}} x_{i_{1} i_{2} i_{3} \ldots i_{I}} \geq 1, \quad \forall i_{I} \\
\sum_{i_{1}=1}^{N_{1}} \sum_{i_{2}=1}^{N_{2}} \sum_{i_{3}=1}^{N_{3}} \cdots \sum_{i_{I}=1}^{N_{I}} x_{i_{1} i_{2} i_{3} \ldots i_{I}}=N
\end{array}\right.
$$

where $c_{i_{1} i_{2} i_{3} \ldots i_{I}}$ is the cost of matching point $p_{i_{1}}^{1}$ to points $\left\{p_{i_{2}}^{2}, p_{i_{3}}^{3}, \ldots, p_{i_{I}}^{I}\right\}$ and $x_{i_{1} i_{2} i_{3} \ldots i_{I}}$ is a binary variable deciding the correctness of the match $\left\langle i_{1}, i_{2}, \ldots, i_{I}\right\rangle$. Since we use inequalities in the constraints (2) to handle the occurrence of hidden seeds, a point can be chosen more than once in an image. The last equality forces the total number of seeds to be $N$.

Let $N^{\prime}=N_{1} N_{2} \ldots N_{I}$, and $\boldsymbol{x}, \boldsymbol{c} \in \mathbb{R}^{N^{\prime}}$ be vector forms of $x_{i_{1} i_{2} \ldots i_{I}}$ and $c_{i_{1} i_{2} \ldots i_{I}}$, respectively. Let $\mathbf{M}$ be a matrix form of (2) except for the last equation. Then the EAP (11)-(2) can be formulated as the following integer program:

$$
P: \min _{\boldsymbol{x} \in \mathcal{C}} \boldsymbol{c}^{\mathrm{t}} \boldsymbol{x}
$$

with the constraint set $\mathcal{C}=\left\{\boldsymbol{x}: \mathbf{M} \boldsymbol{x} \geq[1, \ldots, 1]^{\mathrm{t}}, \boldsymbol{x}^{\mathrm{t}}[1, \ldots, 1]^{\mathrm{t}}=N, x_{\ell} \in\{0,1\}\right\}$, where $x_{\ell}$ is the $\ell$ th element of $\boldsymbol{x}$. Since the value of $x_{\ell}$ is either 0 or 1 and there must be $N$ 1's, an optimal solution of (3) can be considered as choosing $N$ cost coefficients such that the cost is minimized while the constraint $\mathcal{C}$ is satisfied.

\subsection{Dimensionality Reduction of EAP}

Since the EAP has more feasible solutions than the 3DAP, it is not currently possible to solve (1) within a clinically acceptable time. In this paper, we use the same dimensionality reduction approach to the previous work [9], utilizing the following observation: the optimal solution has a near-zero cost when the c-arm pose error is low (it is zero when the pose is exactly known). This feature and Lemma 1 in 9 ] yield a following equivalent problem of reduced dimensionality (for proof, see [9, Sec. 2.2]):

$$
\tilde{P}: \min _{\tilde{\boldsymbol{x}} \in \tilde{\mathcal{C}}} \tilde{\boldsymbol{c}}^{\mathrm{t}} \tilde{\boldsymbol{x}}
$$

where $\tilde{\boldsymbol{x}}, \tilde{\boldsymbol{c}} \in \mathbb{R}^{K}\left(K \leq N^{\prime}\right)$ and $\tilde{\mathcal{C}}=\left\{\tilde{\boldsymbol{x}}: \tilde{\mathbf{M}} \tilde{\boldsymbol{x}} \geq[1, \ldots, 1]^{\mathrm{t}}, \tilde{\boldsymbol{x}}^{\mathrm{t}}[1, \ldots, 1]^{\mathrm{t}}=\right.$ $\left.N, \tilde{x}_{\ell} \in\{0,1\}\right\}$ with $\tilde{\mathbf{M}}=\mathbf{M R}$ and where $\mathbf{R}$ is the dimensionality reduction matrix of size $N^{\prime} \times K$ such that $\left[\begin{array}{llllll}x_{i 1} & 0 & x_{i 2} & 0 & \ldots & x_{i K}\end{array}\right]^{\mathrm{t}}=\mathbf{R}\left[\begin{array}{llll}\tilde{x}_{1} & \tilde{x}_{2} & \ldots & \tilde{x}_{K}\end{array}\right]^{\mathrm{t}}$.

Given a solution $\tilde{\boldsymbol{x}}^{*}$ to the reduced problem $\tilde{P}$, the optimal solution to the original problem $P$ is simply given by $\boldsymbol{x}^{*}=\mathbf{R} \tilde{\boldsymbol{x}}^{*}$.

\subsection{Cost Coefficients and Seed Reconstruction}

To compute $\mathcal{C}$, we need to compute the $3 \mathrm{D}$ intersection of the corresponding lines in space. Due to various errors (e.g., c-arm pose error, seed segmentation error, etc.), these straight lines never intersect, forcing us to compute a symbolic $3 \mathrm{D}$ intersection point. This point is typically defined as the global minimum of an error function. In this paper, we use reconstruction accuracy (RA) based on 
the $L_{2}$ norm of Euclidean distance from the intersection point to the lines as a cost function. By using the RA cost, we can analytically compute a symbolic intersection point using a few summations followed by a $3 \times 3$ matrix inversion 3 . Once the EAP is solved, 3D locations of the $N$ implanted seeds can be determined by the symbolic intersection points used to compute the $N$ chosen RA costs in the solution (those that correspond to 1's in $\boldsymbol{x}$ ).

The dimensionality reduction approach described in Section 2.2 requires only the computation of $K$ cost coefficients that are lower than a threshold. This implies that the exact value of most of the cost coefficients is not required. An efficient way to tell if a cost coefficient is higher than the dimensionality reduction threshold would allow us to skip its exact computation. This unnecessary cost computation can be avoided by utilizing the following Lemma.

Lemma 1. Let $I$ be the total number of $3 D$ lines, and $l_{i}$ and $p_{i}$ be the unit direction vector of line $i$ and a point on the line $i$, respectively. Every $R A$ cost coefficient has the following lower bound:

$$
2 I(I-1) R A \geq \sum_{i_{1}, i_{2} \in\{1,2, \ldots, I\}, i_{2}>i_{1}} d\left(l_{i_{1}}, l_{i_{2}}\right)^{2}
$$

where $R A$ is defined as $R A \triangleq 1 / I \sum_{i=1}^{I} \|\left(P_{I}-p_{i}\right) \times\left. l_{i}\right|^{2}$ and $d\left(l_{i_{1}}, l_{i_{2}}\right)$ is the Euclidean distance of line $i_{1}$ to line $i_{2}$ and with I images.

Proof. Due to the lack of space, the proof is not detailed.

Based on Lemma 1] we propose the following pruning algorithm.

\section{Pruning algorithm for efficient computation of RA costs:}

1: Compute every possible $d\left(l_{i_{1}}, l_{i_{2}}\right)^{2}$ for $I$ images.

2: For the first $i$ images, we have $\tilde{c}_{i_{1}, i_{2}, \ldots, i_{i}}=\sum_{i_{1}, i_{2} \in\{1,2, \ldots, i\}, i_{2}>i_{1}} d\left(l_{i_{1}}, l_{i_{2}}\right)^{2}=$ $\tilde{c}_{i_{1}, \ldots, i_{i-1}}+\sum_{i_{1}=1}^{i-1} d\left(l_{i_{1}}, l_{i}\right)^{2}$. Thus, $\tilde{c}_{i_{1}, i_{2}, \ldots, i_{i}}$ increases as the number of image $i$ increases. When $\tilde{c}_{i_{1}, i_{2}, \ldots, i_{i}}>\eta$, the computation of $\tilde{c}_{i_{1}, i_{2}, \ldots, i_{I}}$ is not required and a large family of cost coefficients can be pruned. This favorable property allows for a recursive algorithm where images are virtually added one at a time and where a list of coefficients lower than $\eta$ is updated.

3: Compute the RAs for indexes $\left(i_{1}, i_{2}, \ldots, i_{I}\right)$ remaining from step 2.

More RA cost coefficients are actually computed from the indexes of coefficients $\tilde{c}_{1,2, \ldots, I}$ lower than the dimensionality reduction threshold $\eta$, than are strictly required because (5) is only an inequality. The performance of the pruning algorithm directly depends on the ratio of the number of RA cost coefficients computed and the number of those actually lower than $\eta$. In practice, we observed that this ratio is in the range of 3 to 15 , which is very compelling, due to its low cost.

\subsection{Linear Programming}

Based on the proposed dimensionality reduction, we solve our reduced integer program (4) using linear programming. We have implemented the linear program for the EAP using MATLAB command linprog followed by a test to confirm that its solution is binary (up to numerical errors). 


\section{$3 \quad$ Numerical Results}

The algorithm was implemented using MATLAB 7.1 on a Pentium4 $2.92 \mathrm{GHz}$ PC with 3.8GB RAM. For simulations, we assumed that the camera parameters of the c-arm were known. For phantom and clinical datasets, we calibrated the carm prior to the procedure and computed the c-arm pose using a tracking fiducial that provides accuracy of $0.33^{\circ}$ in rotation and $0.56 \mathrm{~mm}$ in translation [10].

\subsection{Simulations}

We performed simulation studies using synthetic projection images. We considered four cases with clinically realistic seed density of 2 and 2.5 seeds/cc and prostate size of 35 and $45 \mathrm{cc}$, resulting in 72, 84, 96, and 112 seeds. For each case, we generated three datasets. We generated six projection images on a $10^{\circ}$ cone along the AP-axis in each dataset. In each image, there were $1.7 \%$ on average and up to $5.6 \%$ hidden seeds. We added random error to the pose, uniformly distributed on $[-h, h]$ (reported as $h$ error). Rotation errors varied from $0^{\circ}$ to $4^{\circ}$, with $1^{\circ}$ steps and translation errors varied from $0 \mathrm{~mm}$ to $10 \mathrm{~mm}$, with $2 \mathrm{~mm}$ steps. We exploited the fact that translation errors in depth are always significantly greater than those parallel to the x-ray image plane [3]. For each error type, $240\left(4\right.$ cases $\times 3$ datasets $\left.\times C_{3}^{6}\right)$ and 180 ( 4 cases $\times 3$ datasets $\left.\times C_{4}^{6}\right)$ reconstructions were computed using three and four images, respectively. Shown in Fig. 1, our results imply that the EAP algorithm reliably finds the correct match and reconstruct the seeds with $>95 \%$ accuracy with up to $2^{\circ}$ rotation error and $4 \mathrm{~mm}$ translation error even when only 3 images were used. When the c-arm pose is exactly known, the algorithm can localize the seeds with detection accuracy of $>99 \%$ and reconstruction error $<0.35 \mathrm{~mm}$. We also conducted robustness tests on calibration errors varying from 0 to $10 \mathrm{~mm}$ and angular capture range

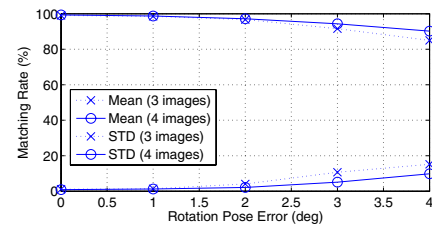

(a)

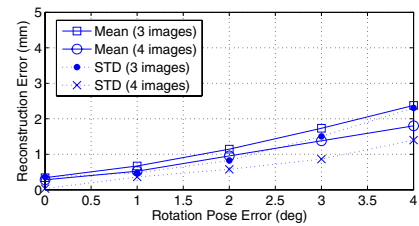

(c)

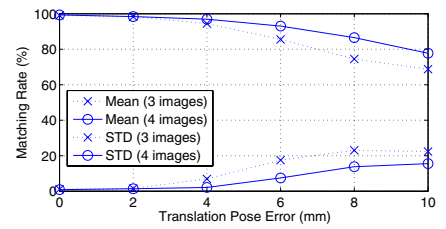

(b)

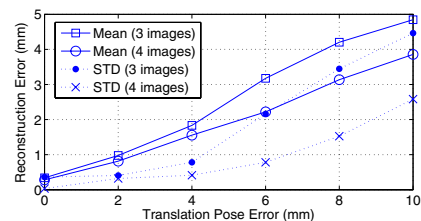

(d)

Fig. 1. Matching rates and reconstruction errors as functions of (a, c) rotation and (b, d) translation pose errors 
Table 1. Phantom experiment results

\begin{tabular}{|c|c|c|c|}
\hline $\begin{array}{c}\text { Number } \\
\text { of seeds }\end{array}$ & $\begin{array}{c}\text { Mean match } \\
\text { rate }(\%)\end{array}$ & $\begin{array}{c}\text { Mean } \pm \text { STD } \\
\text { reconstruction error }(\mathrm{mm})\end{array}$ & $\begin{array}{c}\text { Mean computation } \\
\text { time (s) }\end{array}$ \\
\hline 40 & 97.9 & $0.9 \pm 0.7$ & 1.1 \\
\hline 55 & 100.0 & $0.6 \pm 0.3$ & 2.6 \\
\hline 70 & 99.3 & $0.8 \pm 0.3$ & 3.9 \\
\hline 85 & 97.6 & $1.1 \pm 0.6$ & 6.2 \\
\hline 100 & 99.9 & $1.2 \pm 0.5$ & 10.8 \\
\hline
\end{tabular}

varying from 5 to 25 degrees. Due to the limited space, we do not include the resulting plots, but our method is very robust to the calibration errors and small image acquisition angles achieving seed detection rate of $>99 \%$.

\subsection{Phantom Experiments}

We evaluated the EAP algorithm on a precisely fabricated seed phantom assuring ground-truth. There were five datasets with 40, 55, 70, 85, and 100 implanted seeds (length of $4.9 \mathrm{~mm}$ and diameter of $0.8 \mathrm{~mm}$, similar size to ${ }^{125} \mathrm{I}$ seeds) keeping seed density constant at 1.56 seeds/cc. For each dataset, we acquired six images within a $20^{\circ}$ cone around the AP-axis using a Philips Integris V3000. On average, $5.5 \%$ and up to $22.5 \%$ of the seeds were hidden in each image. The EAP algorithm used 20 combinations of three images from the six available images in each dataset. It successfully localized the seeds with mean accuracy of $\geq 97.6 \%$ and the mean reconstruction error of $\leq 1.2 \mathrm{~mm}$ within about 10 seconds or less depending on the number of seeds. Table 1 summarizes the results and Fig. 2(a) shows a phantom image example with re-projection of the detected seeds.

\subsection{Clinical Experiments}

We validated the EAP algorithm on six patient datasets. For each patient, we acquired two sets of images during the procedure, and one set of images at the end of the procedure using an $O E C 9800$ fluoroscope. The image acquisition angle was about $20^{\circ}$ around the AP-axis. Various number of ${ }^{103} \mathrm{Pd}$ seeds with length of $4.5 \mathrm{~mm}$ and radius of $0.8 \mathrm{~mm}$ were implanted (radio-opaque size of the $\mathrm{x}$-ray marker is about three times smaller than the seed size). There were $0.9 \%$ on average and up to $7.8 \%$ hidden seeds in each image. Since we did not know the exact locations of the seeds, we visually assessed the correspondence between the projection of the estimated seeds and the actual seeds in the images and computed projection errors. The EAP algorithm almost perfectly localized the seeds using 3 images in all cases with mean projection error less than $1 \mathrm{~mm}$ within 10 seconds. We conservatively classified two seeds as mismatched, because the projection of them look matched in some images but look ambiguous in others. Thus, the true detection rate is between $98.1 \%$ and $100 \%$ - in either case, it is a clinically excellent performance. The reconstruction results are shown in Table 2 and Fig. 2(b) shows a fluoroscopy image example with re-projection of the detected seeds. 
Table 2. Clinical experiment results

\begin{tabular}{|c|c|c|c|c|}
\hline $\begin{array}{c}\text { Patient } \\
\text { ID }\end{array}$ & $\begin{array}{c}\text { Number } \\
\text { of seeds }\end{array}$ & $\begin{array}{c}\text { Match } \\
\text { rate }(\%)\end{array}$ & $\begin{array}{c}\text { Mean } \pm \text { STD } \\
\text { projection error }(\mathrm{mm})\end{array}$ & $\begin{array}{c}\text { Computation } \\
\text { time }(\mathrm{s})\end{array}$ \\
\hline \multirow{3}{*}{1} & 22 & 100 & $0.5 \pm 0.3$ & 1.1 \\
\cline { 2 - 5 } & 44 & 100 & $0.7 \pm 0.4$ & 2.2 \\
\cline { 2 - 5 } & 66 & 100 & $0.4 \pm 0.2$ & 4.8 \\
\hline \multirow{3}{*}{2} & 39 & 100 & $0.3 \pm 0.2$ & 2.5 \\
\cline { 2 - 5 } & 82 & 100 & $0.3 \pm 0.3$ & 7.4 \\
\cline { 2 - 5 } & 84 & 100 & $0.9 \pm 0.5$ & 8.7 \\
\hline \multirow{3}{*}{3} & 33 & 100 & $0.2 \pm 0.1$ & 1.4 \\
\cline { 2 - 5 } & 67 & 100 & $0.6 \pm 0.5$ & 3.5 \\
\cline { 2 - 5 } & 70 & 100 & $0.6 \pm 0.6$ & 4.7 \\
\hline \multirow{3}{*}{4} & 35 & 100 & $0.9 \pm 0.5$ & 1.5 \\
\cline { 2 - 5 } & 68 & 100 & $0.2 \pm 0.1$ & 5.8 \\
\cline { 2 - 5 } & 77 & 98.7 & $0.5 \pm 0.3$ & 1.0 \\
\cline { 2 - 5 } & 24 & 100 & $0.6 \pm 0.6$ & 2.4 \\
\cline { 2 - 5 } & 48 & 100 & $0.8 \pm 0.6$ & 3.6 \\
\hline \multirow{3}{*}{6} & 33 & 98.1 & $0.6 \pm 0.5$ & 7.9 \\
\cline { 2 - 5 } & 61 & 100 & $0.3 \pm 0.4$ & \\
\cline { 2 - 5 } & 66 & 100 & $0.1 \pm 0.1$ & 2.4 \\
\hline
\end{tabular}

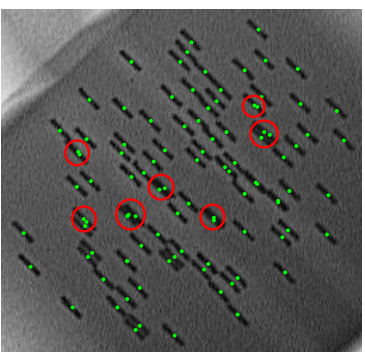

(a)

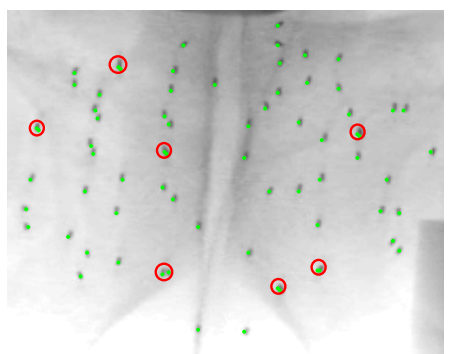

(b)

Fig. 2. Fluoroscopy images with the re-projection of the estimated seed centroids (green dots). Red circles indicate overlapping seeds. (a) Phantom image with 100 seeds. (b) Patient image with 70 seeds.

\section{Conclusion}

We developed a computationally efficient and clinically feasible seed-matching algorithm for prostate brachytherapy seed localization. It automatically resolves the correspondence of seeds from multiple projection images with hidden seeds.

Simulation results imply that the EAP algorithm is robust to realistic carm pose errors with clinically acceptable accuracy. Especially in the phantom study, we used seeds that have similar radio-opaque size to ${ }^{125} \mathrm{I}$ seeds (about three times larger than that of ${ }^{103} \mathrm{Pd}$ seeds), thus creating more hidden seeds (up to 22.5\%). On average, the EAP algorithm was able to correctly find the correspondence with matching rate of $\geq 97.6 \%$. Even in the worst case where $22.5 \%, 7.5 \%$, and $10.0 \%$ of the seeds are hidden in three images, it still achieved $92 \%$ correct matching rate. For clinical datasets, only ${ }^{103} \mathrm{Pd}$ seeds were used, thus having smaller number of hidden seeds than the phantom $\left({ }^{125} \mathrm{I}\right.$ seeds). 
The performance was almost perfect for all 18 datasets, and only two seeds were mismatched in two cases. Small projection errors $(<1.0 \mathrm{~mm})$ imply the accuracy of our reconstruction. Note that a performance better than $97 \%$ detection rate and $2 \mathrm{~mm}$ reconstruction accuracy is considered to be clinically excellent.

The computation time was significantly reduced by adopting a new pruning method for efficient computation of the RA cost. Compared to the previously developed dimensionality-reduction-based algorithm 9] which solves the 3DAP in about 100 seconds, the EAP algorithm is more than 10 times faster and can solve the hidden seed problem within about 10 seconds. This is comparable to MARSHAL [3, one of the fastest seed-matching algorithms in the literature (and it solves an approximate formulation leading to a suboptimal solution).

Finally, we note that although the EAP algorithm is formulated for any number of images, simulation, phantom, and clinical experiment results show that three images are sufficient to achieve clinically adequate outcome in terms of accuracy, robustness, and computation time.

\section{References}

1. Altschuler, M.D., Kassaee, A.: Automated matching of corresponding seed images of three simulator radiographs to allow 3D triangulation of implanted seeds. Phys. Med. Biol. 42, 293-302 (1997)

2. Narayanan, S., Cho, P., Marks, R.: Fast cross-projection algorithm for reconstruction of seeds in prostate brachytherapy. Med. Phys. 29, 1572-1579 (2002)

3. Jain, A.K., Zhou, Y., Mustafa, T., Burdette, E.C., Chirikjian, G.S., Fichtinger, G.: Matching and reconstructoin of brachytherapy seeds using the hungarian algorithm (MARSHAL). Med. Phys. 32, 3475-3492 (2005)

4. Su, Y., Davis, B.J., Herman, M.G., Robb, R.A.: Prostate brachytherapy seed localization by analysis of multiple projections: Identifying and addressing the seed overlap problem. Med. Phys. 31, 1277-1287 (2004)

5. Narayanan, S., Cho, P.S., Marks II, R.J.: Three-dimensional seed reconstruction from an incomplete data set for prostate brachytherapy. Phys. Med. Biol. 49, 34833494 (2004)

6. Su, Y., Davis, B.J., Furutani, K.M., Herman, M.G., Robb, R.A.: Prostate brachytherapy seed reconstruction using adaptive grouping technique. Med. Phys. 34(7), 2975-2984 (2005)

7. Tutar, I.B., Managuli, R., Shamdasani, V., Cho, P.S., Pathak, S.D., Kim, Y.: Tomosynthesis-based localization of radioactive seeds in prostate brachytherapy. Med. Phys. 30, 101-109 (2003)

8. Lam, S.T., Cho, P.S., MarksII, R.J., Narayanan, S.: Three-dimensional seed reconstruction for prostate brachytherapy using hough trajectories. Phys. Med. Biol. 49(4), 557-569 (2004)

9. Labat, C., Jain, A.K., Fichtinger, G., Prince, J.L.: Toward optimal matching for 3D reconstruction of brachytherapy seeds. In: Ayache, N., Ourselin, S., Maeder, A. (eds.) MICCAI 2007, Part II. LNCS, vol. 4792, pp. 701-709. Springer, Heidelberg (2007)

10. Jain, A.K., Mustafa, T., Zhou, Y., Burdette, C., Chirikjian, G.S., Fichtinger, G.: FTRAC - a robust fluoroscope tracking fiducial. Med. Phys. 32, 3185-3198 (2005) 